\title{
Comparação da linfocintilografia com dextrano 500 com a do fitato na pesquisa do linfonodo sentinela no câncer de mama
}

\author{
Lymphoscintigraphy imaging study for sentinel node mapping, comparing dextran 500 with \\ phytate, in breast cancer patients
}

Nilton Leite Xavier ${ }^{1}$, Paulo Ricardo Masiero ${ }^{2}$, Bernardo Leão Spiro ${ }^{3}$, Maria Fernanda Detanico ${ }^{4}$, Ana Lúcia Acosta Pinto ${ }^{5}$, Maria Janilde de Almeida ${ }^{6}$, Carlos Henrique Menke ${ }^{7}$, Jorge Villanova Biazús ${ }^{8}$

\section{RESUMO}

Objetivo: comparar a acurácia de dois radiocolóides na marcação do linfonodo sentinela (LNS) por imagem. Métodos: as pacientes foram incluídas no período de maio de 2002 a abril de 2004. Neste estudo duplo-cego, a paciente foi submetida duas vezes ao mesmo exame, mas com fármacos diferentes, sendo que os fármacos, tecnécio-99m-dextrano 500 (dextrano) e tecnécio-99m-fitato (fitato), foram injetados, na mama, em quatro pontos na área peritumoral e no subcutâneo superficialmente ao tumor, com volume de $2 \mathrm{~mL}$, contendo de 1,0 a 1,5 $\mathrm{mCi}$, em alíquotas de $0,4 \mathrm{~mL}$. Para a obtenção das imagens, duas horas após a injeção do radiofármaco, usamos gama-câmera com colimador de alta resolução. A drenagem linfática axilar foi identificada em imagens radiográficas estáticas, anterior e lateral. A estatística para pares discordantes foi realizada pelo teste de MacNemar e pelo teste Z para proporções. Resultados: na análise das 40 pacientes, obtiveram-se 15 pares com imagens positivas iguais, 4 pares com imagens negativas e 21 pares com imagens distintas, seja porque uma era negativa, seja porque o número de LNS marcados era diferente. A análise do desempenho quanto ao sucesso e insucesso mostrou 35 e 27 imagens positivas e 5 e 13 imagens negativas, respectivamente para o dextrano e o fitato, sendo que das negativas 4 eram comuns. $\mathrm{O}$ estudo estatístico pelo teste de MacNemar mostrou $\mathrm{p}=0,026$, com odds ratio $(\mathrm{OR})=0,11$ e IC $95 \%$ $0,01<\mathrm{OR}<0,85$. A taxa de sucesso foi $67,5 \%$ para o fitato e $87,5 \%$ para o dextrano 500 e a avaliação da acurácia pelos percentuais também foi significante, com $\mathrm{p}=0,032$. Pela ANOVA o número de LNS apontados, foi altamente significante, com $\mathrm{p}=0,008$. Conclusão: este estudo demonstra que a linfocintilografia com dextrano 500 indica linfonodos com mais freqüência que o fitato, quando usados com a mesma metodologia, na obtenção da imagem do LNS de tumores da mama.

PALAVRAS-CHAVE: Cintilografia; Linfonodo/cintilografia; Linfonodo sentinela; Neoplasias mamárias

\section{ABSTRACT}

Purpose: a case-control study comparing two radiocolloids used in scintigraphy to map the sentinel lymph nodes (SLN) in breast cancer patients. Methods: forty patients were prospectively enrolled between May 2002 and April 2004, after signing an informed consent form. In the present double-blind study, each patient was submitted twice to the same examination, a mammary scintigraphy, one with ${ }^{99 \mathrm{~m}} \mathrm{Tc}$-dextran 500 (dextran) and the other with ${ }^{99 \mathrm{~m}} \mathrm{Tc}$-phytate (phytate), on different days. A volume of $2 \mathrm{~mL}$ with $1-1.5 \mathrm{mCi}$ of each radiopharmaceutical, in divided aliquots, was injected in the breast parenchyma in four points around in the tumor and the subcutaneous area superficial to the tumor. The image was obtained $2 \mathrm{~h}$ after the injection, using a gamma camera with high-resolution collimator. The lymph nodes were identified by anterior and lateral static scintigraphic images. Statistical analysis was done with the use of McNemar and Z tests. Results: in the analysis of the 40 patients, we had 15 pairs with positive identical images, 4 pairs with negative images and 21 pairs with inconsistent

1 Professor Adjunto do Departamento de Ginecologia e Obstetrícia (DGO) da Faculdade de Medicina (FAMED) da Universidade Federal do Rio Grande do Sul UFGRS - Porto Alegre (RS) - Brasil.

2 Especialista em Medicina Nuclear do Hospital de Clínicas de Porto Alegre

3 Professor Adjunto do Departamento de Radiologia/ FAMED/ UFRGS

4 Acadêmica da FAMED/ UFRGS. Bolsista de Iniciação Científica, Fundação de Amparo à Pesquisa do Estado do Rio Grande do Sl (FAPERGS);

5 Física do Serviço de Medicina Nuclear do Hospital de Clínicas de Porto Alegre Hospital das Clínicas de Porto Alegre - HCPA - Porto Alegre (RS) - Brasil.

6 Bioquímica do Serviço de Medicina Nuclear do Hospital das Clínicas de Porto Alegre - HCPA - Porto Alegre (RS) - Brasil.

7 Professor Adjunto do Departamento de Ginecologia e Obstetrícia/FAMED/ UFRGS

8 Professor Assistente do Departamento de Ginecologia e Obstetricia/FAMED/ UFRGS

Local de realização do trabalho: Serviços de Medicina Nuclear e de Mastologia do Hospital de Clínicas de Porto Alegre

Apoio financeiro do Fundo de Incentivo à Pesquisa e Eventos (FIPE/ HCPA) e Fundação de Amparo à Pesquisa do Estado do Rio Grande do Sul (FAPERGS).

Correspondência: Nilton Leite Xavier

Rua João Paetzel, 551 - Chácara das Pedras - 91330-280 - Porto Alegre - RS - e-mail: dr_xavier@terra.com.br

Recebido em: 25/8/2004

Aceito com modificações em: 5/6/2005

RevBras Ginecol Obstet. 2005;27(6):340-6 
images, either because one of them was negative, or because the SLN numbers were different. When the protocol was opened, we found 35 and 27 positive images and 5 and 13 negative images for dextran and phytate treatment groups, respectively. Among the negative images, 4 were shared by both groups. The McNemar test, used for the statistical analysis, showed $\mathrm{p}=0.026$, odds ratio $(\mathrm{OR})=0.11$ with $95 \% \mathrm{CI} 0.01<\mathrm{OR}<0.85$. The accuracy, evaluated by the success ratio of the SLN mapping, was $67.5 \%$ for phytate and $87.5 \%$ for dextran, with $\mathrm{p}=0.032$. Analysis of variance of the SLN number in lymphoscintigraphy images showed $\mathrm{p}=0.008$. Conclusion: these results recommend the use of dextran instead of phytate for the SLN study of breast carcinoma by scintigraphy, when the same methodology is being used.

KEYWORDS: Radionuclede imaging; Lymph nodes/radionuclede imaging; Sentinel lymph node; Breast neoplasms

\section{Introdução}

O estudo dos linfonodos axilares no pré-operatório por meio do exame clínico, mamografia ou ultra-sonografia pode trazer informações a respeito do tamanho, porém não são métodos sensiveis para a definição do comprometimento metastático regional, tendo esta informação importante valor prognóstico $^{1-3}$. Neste sentido, tem sido pesquisado o valor da detecção do linfonodo sentinela (LNS), mantendo o prognóstico do estadiamento axilar e associado a menor mutilação, evitando-se o esvaziamento completo da axila ${ }^{4-6}$. O estadiamento linfático regional, tradicionalmente realizado pela dissecção axilar linfonodal, está sendo substituído pela biópsia seletiva do primeiro linfonodo de drenagem, na rota de disseminação do câncer de mama, isto é, do $\mathrm{LNS}^{7,8}$. Isto ocorre porque, apesar de a maioria dos espécimes dissecados não conter metástases, a dissecção axilar linfonodal é responsável por mais morbidade e custos do que o próprio tratamento cirúrgico do tumor primário $^{2,3,6,8,9}$. Há uma década a biópsia do LNS está sendo usada e os estudos correlacionando a biópsia com a dissecção axilar mostraram taxa de sucesso de $88 \%$, sensibilidade de $93 \%$ e acurácia de $97 \%{ }^{10}$.

A terceira circulação, constituída pelo sistema linfático, tem sido estudada na sua anatomia e fisiologia, porque a linfocintilografia tem aplicação clínica em várias especialidades, e tem merecido muitos estudos, com o reconhecimento da utilidade do LNS em vários tipos de câncer ${ }^{11-13}$. No mapeamento linfático da axila, os estudos têm mostrado a constância dessa drenagem, com resultados linfocintilográficos reprodutiveis ${ }^{10,12}$. Um estudo $^{10}$ com pequeno grupo de pacientes, usando um radiocolóide com partículas com diâmetro inferior a $80 \mathrm{~nm}$, com dose de $3 \mathrm{mCi}$ e injeção intratumoral, mostrou imagens semelhantes às do dia seguinte, quando se repetiu o mesmo procedimento.

A linfocintilografia mamária antes da abordagem cirúrgica, com a localização do LNS, prediz sucesso na biópsia do $\mathrm{LNS}^{9,10}$. Qual fármaco apresenta a melhor captação pelo linfonodo de drenagem ainda está por ser definido ${ }^{11,14-18}$. É também controverso qual a concentração do fármaco, qual o volume a ser injetado e qual o local da injeção do radiocolóide $6-8,18-21$. A injeção subcutânea de radiopartículas tem permitido a visualização de um ou mais linfonodos axilares, mas esta via não permite a visualização do LNS porventura existente na cadeia mamária interna ${ }^{20}$. A injeção no parênquima mamário, em torno do tumor, deve ser usada quando o propósito é determinar o estádio de forma tão acurada quanto possivel e para identificar só $\mathrm{LNS}^{20}$.

Experiência anterior deste grupo com o tecnécio (Tc)-99m-dextrano 500 foi publicada ${ }^{4,22}$ e decidimos estudar a eficácia deste radiofármaco comparada com a do Tc-99m-fitato porque não há estudo comparativo entre estas partículas, de tamanhos equivalentes e $\mathrm{pH}$ distintos.

\section{Métodos}

As pacientes foram recrutadas prospectivamente no periodo de maio de 2002 a abril de 2004, no ambulatório de Mastologia do HCPA e na clínica de um dos pesquisadores. Todas tinham o diagnóstico histológico, obtido por biópsia por fragmento com agulha grossa, de câncer de mama, com tumores de até $5,0 \mathrm{~cm}$, ainda não tratadas cirurgicamente e com a axila clinicamente negativa. Foram excluídas as pacientes com doença sistêmica, tumores múltiplos, as grávidas e as submetidas à quimioterapia neoadjuvante. $\mathrm{Na}$ amostra de 42 pacientes, duas não foram submetidas ao protocolo. A média de idade foi 55 anos, o indice de massa corporal (IMC) foi de 26,9 e o tamanho médio dos tumores foi $2,0 \mathrm{~cm}$. Após assinarem o consentimento informado, previamente aprovado pela Comissão de Ética em Pesquisa do HCPA, foram randomizadas para o estudo duplocego, que comparou a eficácia de dois radiofármacos, o fitato e o dextrano, cujas características técnicas foram informadas pelo Instituto de Pesquisas Nucleares (IPEN), SP. Segundo o IPEN, o diâmetro da grande maioria das partículas do dextrano tem entre 10 e 15 nanômetros, num $\mathrm{pH}$ de 3 a 4, e o fitato apresenta tamanho de partículas entre 10 e 17 nanômetros, num $\mathrm{pH}$ de 5 a 6 , sendo solu- 
ções com partículas de tamanhos equivalentes, porém diferentes quanto ao $\mathrm{pH}$.

Os frascos contendo o dextrano e o fitato, que foram usados na pesquisa, foram codificados no Serviço de Medicina Nuclear do Hospital de Clínicas de Porto Alegre. Marcavam-se duas linfocintilografias mamárias para cada paciente, que aleatoriamente usavam ora um ora outro fármaco, conforme a lista de códigos, estando os médicos responsáveis por sua administração e detecção das imagens cegos em relação ao material utilizado. A segunda linfocintilografia foi realizada com o intervalo minimo de 72 horas e máximo de sete dias.

Para a obtenção das imagens usamos gamacâmera com colimador de alta resolução e detector retangular com 48 fotomultiplicadores, cristal com espessura de 9,0 mm e variação de energia de 55$400 \mathrm{keV}$ (GE, Model Millenium; MPR System: Anger Camara). A drenagem linfática foi identificada com imagens cintilográficas estáticas, nas projeções anterior e lateral, obtidas duas horas após a injeção peritumoral e subcutânea de $1,0-1,5 \mathrm{mCi}$ do radiofármaco, contido em $2 \mathrm{~mL}$ da solução e injetado em cinco frações de $0,4 \mathrm{~mL}$ e sem massagem local. A injeção, nas duas ocasiões, era sempre realizada por um dos pesquisadores e da mesma maneira.

Os laudos emitidos pelo serviço de Medicina Nuclear eram simples e informavam a presença ou a ausência de imagem de nódulo, compativel com o LNS, o local da imagem e o número de nódulos visualizados, na imagem obtida duas horas após a injeção do radiofármaco codificado. Não há correlação deste estudo com os resultados cirúrgicos.

Foi avaliado o sucesso e o insucesso de cada um dos radiofármacos quanto à detecção por imagem do LNS e também se fez a avaliação quantitativa. A avaliação do número de linfonodos, pelo escrutínio das imagens, era realizada por dois especialistas e pelo primeiro pesquisador. Obtiveram-se dois grupos. Num deles cada paciente teve suas duas linfocintilografias com imagens semelhantes e idêntico diagnóstico, positivas ou negativas, quanto à presença do linfonodo de drenagem. No outro, cada paciente teve linfocintilografias com imagens diferentes.

Calculou-se, nos 40 pares, o número médio de linfonodos marcados por cada um dos fármacos e usou-se a ANOVA na análise estatística.

Para os pares discordantes, tratando-se de amostras pareadas, usamos o teste de MacNemar. A comparação entre os pares foi de proporção com variável dicotômica, cuja avaliação foi feita com teste $Z$ para proporções. Para os pares em que uma ou as duas imagens foram negativas, avaliou-se a influência da idade das pacientes e do IMC, como variáveis quantitativas, comparando-se a média, numa análise de variância. Na estimativa do tamanho da associação usou-se o intervalo de confiança de 95\% (IC 95\%) e os valores de $\mathrm{p}<0,05$ foram considerados estatisticamente significantes.

\section{Resultados}

Dos quarenta pares, observaram-se $15 \mathrm{com}$ imagens positivas, quatro com imagens negativas e 21 com imagens distintas (Figuras 1 e 2). Constatou-se que dos 21 pares desiguais, havia 10 imagens negativas, sendo apenas uma do dextrano. Nas outras 11 imagens pareadas, havia diferença de número de linfonodos apontados (Figura 2), e o dextrano foi superior em nove delas. Nestes 40 pares, as médias de LNS apontados pelo dextrano e pelo fitato foram 1,5 e 0,9, respectivamente $(p=0,008)$, (Tabela 1$)$.
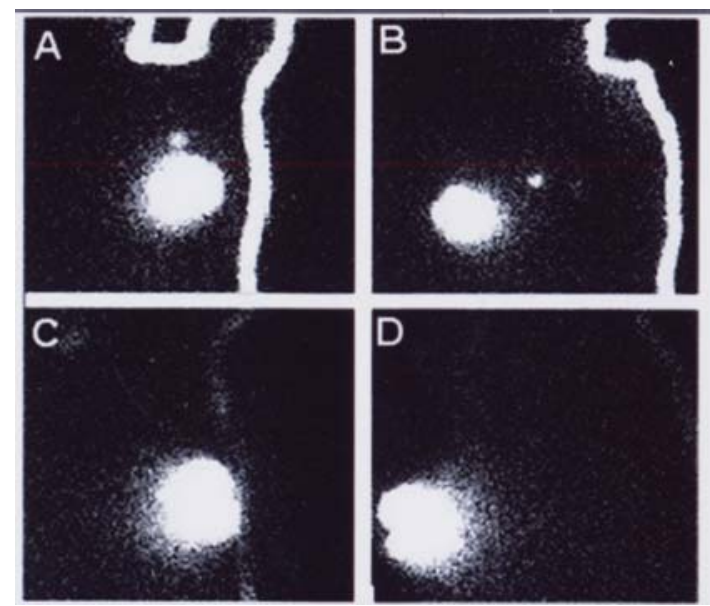

Figura 1 - Imagem $A$ (frente) eB (perfil), com o uso de dextrano, mostrando um linfonodo axila (sucesso). Imagem C e D, idem, com fitato e imagem negativa para linfonodo (insucesso), mostrando apenas o tumor envolvido pelo radiofármaco.
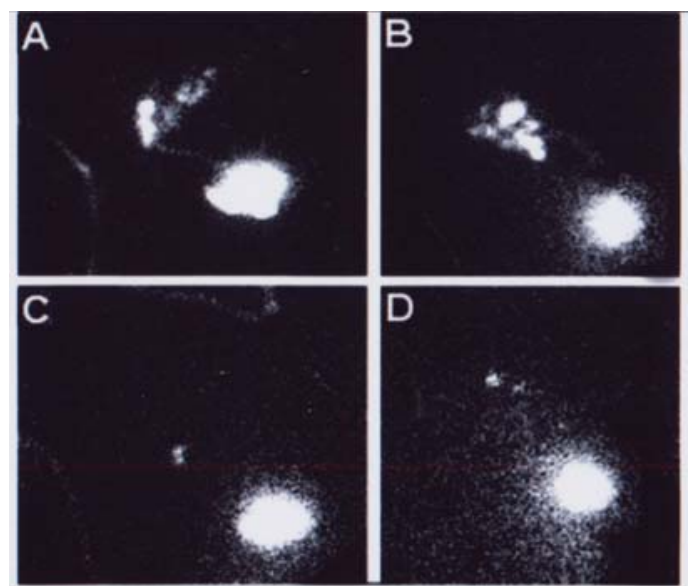

Figura 2 - A (frente) e B (perfil) com Tc-99m-dextrano, que mostra vários linfonodos na axila e percebe-se $o$ trajeto do radiofármaco a partir do tumor até os linfonodos; $C$ e D, idem, apenas 2 linfonodos, com uso de Tc-99m-fitato. Ambos com sucesso, porém diferentes no número de linfonodos apontados. 
Tabela 1 - Desempenho quantitativo: número de linfonodo sentinela (LNS) apontado. Estatística: ANOVA para amostras pareadas, com dextrano mostrando desempenho superior e mostrando em média 1,5 LNS por caso. Estatística para variável quantitativa (ANOVA)

\begin{tabular}{lcccc}
\hline Fármacos & $\mathbf{n}$ & Média & Variância & $\begin{array}{c}\text { ANOVA F } \\
\text { estatístico }\end{array}$ \\
\hline Dextrano & 40 & 1,5 & 1.595 & $\mathrm{~F}=7,29$ \\
Fitato & 40 & 0,9 & 0.583 & $\mathrm{p}=0,008$ \\
\hline
\end{tabular}

$\mathrm{Na}$ avaliação absoluta - sucesso ou insucesso - obtiveram-se 27 e 35 imagens positivas e iguais (Figura 3) para os radiofármacos, 13 imagens negativas para um deles e cinco para o outro, porém quatro delas, negativas (10\%), foram comuns aos dois. Houve $67,5 \%$ de sucesso para o fitato e $87,5 \%$ para o dextrano. A análise, anotada na Tabela 2, mostrou $\chi^{2}$ de MacNemar igual a 4,9, odds ratio 0,11 e $\mathrm{p}=0,026$. Sob esta ótica, a diferença de desempenho, entre os dois fármacos, foi significante.

Tabela 2 - Desempenho absoluto: o sucesso (+) para o dextrano foi de 87,5\% (35 casos) e para o fitato foi de $67,5 \%$ (27 casos); o insucesso (-) para o dextrano ocorreu em apenas 5 casos e para o fitato em 13 casos. Teste estatístico de MacNemar: Odds ratio $=0,11$; intervalo de confiança de $95 \%(0,01-0,85) ; p=0,02$.

\begin{tabular}{lccc}
\hline & Dextrano + & Dextrano - & Total \\
\hline Fitato + & 26 & 1 & 27 \\
Fitato - & 9 & 4 & 13 \\
Total & 35 & 5 & 40 \\
\hline
\end{tabular}

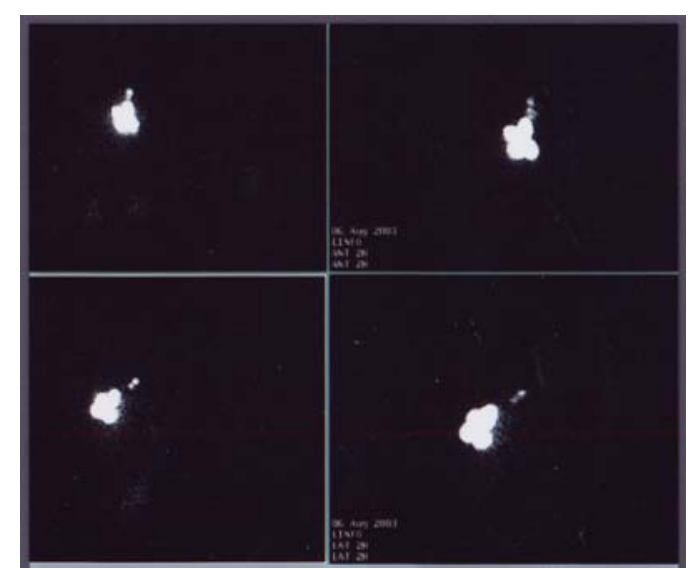

Figura 3 - $A$ e $B$, imagens de frente; $C$ e $D$, imagens laterais. $A$ e $C=$ fitato; $B$ e $D=$ dextrano. Os dois fármacos tiveram sucesso e mostram o mesmo número de linfonodos; são imagens semelhantes com 2 linfonodos justapostos.

Empregando a estatística do $Z$ para proporções, como o dextrano, na comparação dos 40 pares, teve cinco imagens negativas $(12,5 \%)$ e o fitato teve $13(32,5 \%)$, tem-se $\chi^{2}=4,59$ e $p=0,032$, com significância estatística, portanto.
A influência da idade e do IMC, pela análise da média e da variância, no sentido de avaliar qual o motivo da não identificação do LNS, não mostrou diferença ( $p=0,13$ e 0,37 , respectivamente).

Anotou-se a incidência simultânea de imagens do LNS na axila e na cadeia linfática da mamária interna, que foi de $7,5 \%$, com três casos.

\section{Discussão}

A literatura publica resultados obtidos com vários radiofármacos, em volume e dose variada, conforme a pesquisa e a experiência de cada serviço ${ }^{4,9,14,16,21,23}$. Há poucos resultados comparativos. No Brasil dispomos dos marcadores associados ao dextrano e ao fitato, ambos produzidos pelo IPEN. Delineamos um estudo comparativo, que pode contribuir para a escolha do fármaco de mais sucesso. O estudo ${ }^{12}$ que utilizou duas linfocintilografias com o mesmo radiofármaco, porém com $3 \mathrm{mCi}$, concluiu que o método é altamente reprodutivel. Um ponto que merece crítica é o uso de dose alta do fármaco, embora outros estudos ${ }^{24,25}$ considerem que sua concentração favoreça o resultado.

Ao estudar ${ }^{19}$ o desempenho do Tc-99m-rênio (rênio), obtido na Europa, com tamanho de partículas em torno de $100 \mathrm{~nm}$, comparado com o do Tc-99m-fitato, produzido no Japão, com partículas cujo tamanho é desconhecido, mas estimadas como similares às do rênio, alguns vieses foram observados. Não houve uma amostra pareada e a injeção de $1 \mathrm{~mL}$, com rênio, foi dividida em quatro aliquotas nas primeiras 60 pacientes e em cinco aliquotas nas 40 seguintes, semelhante ao nosso estudo. O fitato foi usado a seguir, sendo o uso de cinco aliquotas em 60 pacientes e de 3 alíquotas em 40. A injeção era subcutânea quando feita em três ou quatro aliquotas e a dose do tecnécio variou de 0,4 a 1,5 mCi. Após a injeção, era realizada uma massagem, durante um a dois minutos; algumas imagens eram obtidas após duas horas e outras, operadas no dia seguinte, após 16 horas. $\mathrm{Na}$ análise as pacientes não foram discriminadas adequadamente e formaram apenas dois grupos, segundo o fármaco usado. Os dois tiveram desempenho semelhante, mas o rênio marcou mais nódulos do que o fitato, com média 3,0 e 1,5, respectivamente. Não houve influência da idade, do IMC e do tamanho do nódulo, apesar dos vieses apontados. Em nosso estudo com o fitato marcando em média 0,9 linfonodos e o dextrano 1,5, a diferença foi também significativa.

A taxa de absorção das partículas injetadas é inversamente proporcional ao seu tamanho ${ }^{18}$. 
As partículas com diâmetro menor do que $5 \mathrm{~nm}$ entram principalmente no sistema venoso; as particulas entre 5-25 nm entram nos capilares linfáticos pelos orificios entre as junções celulares e pelos trajetos intercelulares, os quais mesmo fechados têm de 10 a $25 \mathrm{~nm}^{18}$. Para as partículas com mais de 18-20 nm a matriz intersticial de elastina começa a ser uma barreira para o movimento e para sua passagem para os linfáticos ${ }^{17,18}$. Partículas com diâmetro entre 25 e $75 \mathrm{~nm}$ podem entrar no lúmen linfático por pinocitose, sendo que a partir de 50 até $100 \mathrm{~nm}$ têm dificuldade de movimentação através do interstício celular formado de elastina, fibrilas e fibras de colágeno ${ }^{17,18}$. Existem orificios grandes, ocasionais, que se abrem na dependência de efeitos de movimentos e tensão nos tecidos moles. Partículas com diâmetro maior do que $100 \mathrm{~nm}$ entram através de janelas ocasionais que ocorrem na parede endotelial. Para isso são necessários fatores mecânicos que abrem os grandes orificios e que incluem a pressão do fluído intersticial, os movimentos e massagem nos tecidos moles ${ }^{17,18,20,26}$ e, também, a lesão de capilares pela agulha da injeção e as propriedades da superficie das partículas ${ }^{13,27,28}$.

Alguns pesquisadores referem que o fluxo linfático deve ficar o mais próximo de sua fisiologia $^{18,20}$, e interrogam como interpretar as imagens obtidas depois da injeção de grandes volumes, que podem ativar o fluxo linfático e ocasionar falsos trajetos $^{18,20,24}$. A massagem suave aumenta a produção e a taxa do fluxo linfático e a experiência sugere que não causa comprometimento na detecção do $\operatorname{LNS}^{18,20,26}$.

O radiocolóide ideal para o mapeamento linfático, baseado em fatores anatômicos e fisiológicos dos capilares linfáticos e dos linfonodos, teria entre 10 e $25 \mathrm{~nm}^{18}$, com entrada fácil no sistema linfático e também boa retenção nos linfonodos drenados, e o colóide sulfeto de antimônio seria um exemplo ${ }^{18}$.

Nesta pesquisa os dois fármacos experimentados apresentam diferença não significativa no tamanho das partículas, sendo diferentes apenas quanto ao $\mathrm{pH}$. O pH da substância injetada interage com o meio e a pressão oncótica aumenta se ocorrer aumento do $\mathrm{pH}^{28}$. No entanto, sabe-se que também a carga elétrica, a hidrofobia e a presença de ligantes de absorção podem influenciar ${ }^{26,28}$ na absorção do radiocolóide. O tamanho das partículas varia com o $\mathrm{pH}$, porém quanto esta diferença no espaço intersticial e no líquido linfático afeta o tamanho da partícula e a sua captação, no tecido vivo, permanece por ser investigado ${ }^{13}$.

Tanto o dextrano quanto o fitato apresentam partículas no tamanho ideal ${ }^{6,18}$, não necessitando de grande volume nem de massagem local como sói acontecer com as partículas grandes ${ }^{17,25}$. Neste estudo radiofármacos foram analisados tanto no sucesso e insucesso quanto no número de LNS apontados, e o dextrano teve desempenho superior, em quaisquer das análises.

O dextrano é polissacarídeo clinicamente usado como substituto de plasma e é conhecido por permanecer no espaço vascular após a administração intravenosa. O Tc-99m-dextrano 70 tem estabilidade, com captação lenta para outros tecidos e imagens altamente contrastadas dos canais linfáticos. A técnica de preparação do Tc-99m-dextrano 70 é pouco dispendiosa, a partir de intermediários disponiveis comercialmente ${ }^{21}$. O primeiro relato na literatura ${ }^{4}$ sobre o uso de Tc-99m-dextrano 500, para a pesquisa do LNS com o uso do gama-probe ou sonda gama, com $89 \%$ de sucesso, foi similar aos publicados com enxofre coloidal ou albumina, que são, também, produtos muito usados ${ }^{13}$.

Alguns autores confirmam boa taxa de detecção com uso de partículas grandes ${ }^{23}$. O relato do uso de três fármacos ${ }^{23}$, em câncer de mama, com o uso de partículas com 100 nm, 100 a 600 $\mathrm{nm}$ e menores do que $80 \mathrm{~nm}$, todos marcados com 80 a $100 \mathrm{MBq}(2-2,5 \mathrm{mCi})$, mostrou que não houve diferença entre eles, com imagens positivas em $60,9 \%$ em uma hora e $82,8 \%$ em duas horas. Tivemos $7,5 \%$ de imagens, na região correspondente à mamária interna, e outros autores ${ }^{9,23}$ obtiveram de 3 a 9,3\% de imagens simultâneas para o LNS axilar e paraesternal.

O diâmetro das partículas mais usadas varia de 5 a $80 \mathrm{~nm}$, proporcionando adequada migração do sítio da injeção até o LNS ${ }^{13,18}$. Como exemplo temos o Tc-99m-sulfocolóide e o Tc-99m-HSA nanocolóide, com tamanho de partículas cuja média é inferior a 30 e $80 \mathrm{~nm}$, respectivamente. A despeito desse fato, alguns preferem traçadores com partículas grandes, maiores do que $100 \mathrm{~nm}$, para diminuir o número de linfonodos radioativos na axila ${ }^{19,21}$, contudo esta abordagem pode ocasionar subavaliação do número de LNS. Estudos com partículas de tamanhos distintos ${ }^{9,18}$ mostram imagem positiva em $87 \%$ dos casos e média de linfonodos visualizados maior, a favor das partículas menores do que $80 \mathrm{~nm}$.

Os nossos resultados, com $87,5 \%$ de LNS visualizados, são similares aos da literatura, com outros radiofármacos. O uso de altas concentrações de radiofármaco ${ }^{16,17,24}$, na procura de melhor resultado, na nossa experiência, não se justifica. Nesta pesquisa, com dois fármacos com partículas pequenas, as diferenças de desempenho devem ser o resultado da interação do $\mathrm{pH}$ com o meio $^{28}$, da hidrofobicidade ${ }^{28}$, da carga elétrica ${ }^{28}$ e 
da diferença na característica da superficie ${ }^{13,28} \mathrm{e}$ capacidade de opsonização ${ }^{13,27}$ dos colóides, como já referimos anteriormente.

$\mathrm{Na}$ avaliação de fatores dependentes das características das pacientes, que pudessem influenciar nos resultados, verificamos que as pacientes mais idosas, talvez por lipossubstituição do tecido linfóide, têm maior possibilidade de linfocintigrafia negativa. Sato et al. ${ }^{29}$ recomendam cautela na pesquisa do LNS em pacientes idosas, o que reforça a tendência mostrada nesta pesquisa, necessitando-se de estudos adicionais com maior número de pacientes.

Em resumo, comprovamos que, dentre as duas soluções, o dextrano marca mais casos e tem mais linfonodos marcados à cintilografia que a solução de fitato, quando usados em igual volume, mesma técnica de injeção e mesmo tempo entre a injeção e a obtenção da imagem cintilográfica.

\section{Agradecimentos}

Agradecemos ao IPEN, na pessoa da Dra. Constância Pagano Gonçalves da Silva, que nos informou sobre os dados técnicos dos fármacos deste estudo, ao Fundo de Incentivo ao Pesquisador (FIPE/ HCPA), que financiou esta pesquisa, e aos funcionários da Medicina Nuclear, Elaine Maria Lopes dos Santos e Sílvia Cristina Pinto Borges, secretárias, e Stela Mares Fernandes, técnica em radiologia, que contribuiram para o bom êxito deste estudo. Agradecemos ao ginecologista Miguel da Cunha Xavier, que nos encaminhou pacientes, e à FAPERGS, que contribuiu com a Bolsa de Iniciação Científica.

\section{Referências}

1. Kelley MC, Hansen N, McMasters KM. Lymphatic mapping and sentinel lymphadenectomy for breast cancer. Am J Surg. 2004;188(1):49-61.

2. Giuliano AE. Mapping a pathway for axillary staging: a personal perspective on the current status of sentinel lymph node dissection for breast cancer. Arch Surg. 1999;134(2):195-9.

3. Jatoi I, Hilsenbeck SG, Clark GM, Osborne CK. Significance of axillary lymph node metastasis in primary breast cancer. J Clin Oncol. 1999;17(8):233440.

4. Xavier NL, Amaral BB, Cerski CT, Fuchs SC, Spiro BL, Oliveira OL, et al. Sentinel lymph node identification and sampling in women with early breast cancer using 99m-Tc labelled dextran 500 and patent blue V dye. Nucl Med Commun. 2001;22(10):1109-17.

5. Jakub JW, Cox CE, Pippas AW, Gardner M, Pendas $\mathrm{S}$, Reintgen DS. Controversial topics in breast lymphatic mapping. Semin Oncol. 2004;31(3):32432.

6. Uren RF, Howman-Giles RB, Thompson JF. Regarding sentinel lymph node localization in early breast cancer. J Nucl Med. 1999;40(8):1403-6.

7. Krynyckyi BR, Kim CK, Goyenechea MR, Chan PT, Zhang ZY, Machac J. Clinical breast lymphoscintigraphy: optimal techniques for performing studies, image atlas, and analysis of images. Radiographics. 2004;24(1):121-45.

8. Posther KE, Wilke LG, Giuliano AE. Sentinel lymph node dissection and the current status of American trials on breast lymphatic mapping. Semin Oncol. 2004;31(3):426-36.

9. Mariani G, Moresco L, Viale G, Villa G, Bagnasco M, Canavese G, et al. Radioguided sentinel lymph node biopsy in breast cancer surgery. J Nucl Med. 2001;42(8):1198-215.

10. Liberman L. Lymphoscintigraphy for lymphatic mapping in breast carcinoma. Radiology. 2003;228(2):313-5.

11. Paganelli G, De Cicco C, Chinol M. Sentinel node localization by lymphoscintigraphy: a reliable technique with widespread applications. Recent Results Cancer Res. 2000;157(2):121-9.

12. Tanis PJ, Valdes Olmos RA, Muller SH, Nieweg OE. Lymphatic mapping in patients with breast carcinoma: reproducibility of lymphoscintigraphic results. Radiology. 2003;228(2):546-51.

13. Szuba A, Shin WS, Strauss HW, Rockson S. The third circulation: radionuclide lymphoscintigraphy in the evaluation of lymphedema. J Nucl Med. 2003;44(1):43-57.

14. Jinno H, Ikeda T, Matsui A, Kitagawa Y, Kitajima M, Fujii H, et al. Sentinel lymph node biopsy in breast cancer using technetium-99m tin colloids of different sizes. Biomed Pharmacother. 2002;56 Suppl 1:213s-216s.

15. Noguchi M. Current controversies concerning sentinel lymph node biopsy for breast cancer. Breast Cancer Res Treat. 2004;84(3):261-71.

16.Valdes-Olmos RA, Jansen L, Hoefnagel CA, Nieweg OE, Muller SH, Rutgers EJ, et al. Evaluation of mammary lymphoscintigraphy by a single intratumoral injection for sentinel node identification. J Nucl Med. 2000;41(9):1500-6.

17. Wilhem AJ, Mijnhout S, Franssen EJ. Radiopharmaceuticals in sentinel lymph-node detection: an overview. Eur J Nucl Med. 1999;26(4 Suppl):S36-42. 
18. Uren RF, Thompson JF, Howland-Giles RB. Lymphatic drainage of the skin and breast. Amsterdam: Harwood Academic; 1999. p. 29-50.

19. Koizumi M, Nomura E, Yamada Y, Takiguchi T, Makita M, Iwase T, et al. Radioguided sentinel node detection in breast cancer patients: comparison of 99m Tc phytate and 99m Tc rhenium colloid efficacy. Nucl Med Commum. 2004;25(10):1031-7.

20. Tanis PJ, Nieweg OE, Valdes Olmos RA, Kroon BB. Anatomy and physiology of lymphatic drainage of the breast from the perspective of sentinel node biopsy. J Am Coll Surg. 2001;192(3):399-409.

21. Dansereau RN, Line BR. Clinical production of pharmaceutical grade Tc-99m dextran 70 for lymphoscintigraphy. Clin Nucl Med. 2000;25(3):17981.

22.Xavier NL, Amaral BB, Spiro BL, Almeida MJ, Menke $\mathrm{CH}$, Biazú JV, et al. Detecção do linfonodo sentinela em câncer de mama: comparação entre métodos. Rev Bras Ginecol Obstet. 2002;24(7):479-84.

23. Liu TJ, Wang SJ, Tsai SC. Lymphoscintigraphy using larger colloid particles may enhance visualization of the sentinel node in breast cancer: a case report. Clin Nucl Med. 2000;25(3):191-2.
24. Kraft O, Safarcik K, Stepien A. Sentinel lymph node detection and biopsy in breast cancer e malignant melanoma. World J Nucl Med. 2004;3(1):26-32.

25.Valdes Olmos RA, Tanis PJ, Hoefnagel CA, Nieweg OE, Muller SH, Rutgers EJ, et al. Improved sentinel node visualization in breast cancer by optimizing the colloid particle concentration and tracer dosage. Nucl Med Commun. 2001;22(5):579-86.

26. Leidenius MH, Leppanen EA, Krogerus LA, Smitten KA. The impact of radiopharmaceutical particle size on the visualization and identification of sentinel nodes in breast cancer. Nucl Med Commun. 2004;25(3):233-8.

27. Gurfinkel EP. Genética y biología molecular en cardiología (III) infección y aterosclerosis. Rev Cuba Med. 2001;40(4):273-87.

28. Ikomi F, Hanna GK, Schmid-Schonbein GW. Sizeand surface-dependent uptake of colloid particles into the lymphatic system. Lymphology. 1999;32(3):90-102.

29. Sato K, Tamaki K, Shigewaka T, Tsuda H, Kosuda $\mathrm{S}$, Kusano S, et al. Clinicopathologic and technical factors associated with the uptake of radiocolloid by sentinel nodes in patients with breast cancer. Surg Today. 2003;33(6):403-7. 